\title{
Effectiveness of Ring Vaccination as Control Strategy for Ebola Virus Disease
}

\section{Adam J. Kucharski, Rosalind M. Eggo, Conall H. Watson, Anton Camacho, Sebastian Funk, W. John Edmunds}

Using an Ebola virus disease transmission model, we found that addition of ring vaccination at the outset of the West Africa epidemic might not have led to containment of this disease. However, in later stages of the epidemic or in outbreaks with less intense transmission or more effective control, this strategy could help eliminate the disease.

$\mathrm{D}$ uring 2014-2015, trials of candidate vaccines for Ebola virus disease (EVD) were fast tracked in response to the unprecedented EVD epidemic in West Africa (1). In March 2015, a phase 3 ring vaccination trial of a recombinant vesicular stomatitis virus-Zaire Ebola virus vaccine began in Guinea (2). Interim trial results suggested that the vaccine could have a high level of efficacy in humans (3). Ring vaccination has also been used for disease control, notably in the final stages of the smallpox eradication program (4). Furthermore, a recent modeling study calibrated by using population-level EVD data from Sierra Leone and Liberia (5) suggested that ring vaccination could supplement case isolation and contact tracing in reducing transmission. However, it remains unclear whether prompt ring vaccination, as opposed to large-scale mass vaccination, could have contained the EVD epidemic in West Africa, and under what circumstances it could be effective in controlling future outbreaks.

\section{The Study}

We developed a stochastic model of EVD transmission (online Technical Appendix, http://wwwnc.cdc.gov/EID/ article/22/1/15-1410-Techapp1.pdf) using individual-level transmission data from Guinea to inform our model structure. Transmission chains during March-August 2014 suggest substantial variation in the number of secondary cases generated $(6,7)$. In particular, index cases, defined as those that could not be linked to an already known transmission chain, had a reproduction number of $R_{m}=7$, where $m$ indicates missed cases and $R_{m}$ denotes the average number of secondary cases generated, whereas cases within known transmission chains $(w)$ had a reproduction number of $R_{w}=$ 0.66 (online Technical Appendix, Figure 1).

Author affiliations: London School of Hygiene and Tropical

Medicine, London, UK

DOI: http://dx.doi.org/eid2201.151410
In the model, transmission followed a branching process $(8)$, and secondary cases were generated from a negative binomial distribution to include potential for superspreading events $(6,9)$. Each cluster started with an index case, which generated an average of $R_{m}=7$ secondary cases. Many EVD cases reported in Guinea were not part of already known transmission chains (online Technical Appendix, Figure 2). We therefore assumed there was a probability $\rho$ that a secondary case would missed and go on to seed an independent transmission cluster as an index case with $R_{m}=7$. Otherwise, the case would remain within the known chain of transmission (with probability $1-\rho$ ); these cases would then generate an average of $R_{w}=0.66$ secondary cases. The simulated outbreaks ended when, by chance, no secondary cases were generated by active cases. Distributions of incubation period, duration of infectiousness, and time to reporting were obtained from reported values for Guinea in 2014 (10). Model simulations produced similar patterns to those observed in 2014 (Figure 1). When half of the cases were missed, the overall reproduction number, defined as the mean number of secondary cases generated across all infectious persons, was $\approx 1.5$, which was similar to values observed in early 2014 in West Africa (11) and in the initial stages of other outbreaks (online Technical Appendix Table 1).

We simulated ring vaccination by using a protocol similar to that used in Guinea trial (3). We defined a ring as all persons who could potentially form part of the known chain of transmission (i.e., traceable contacts of infected persons within a transmission cluster and their contacts). Once the index case was reported, we assumed it took 2 days to vaccinate a ring and that protective immunity developed 7 days after vaccination. In the model, we assumed that vaccine efficacy was $80 \%$ and that $70 \%$ of the ring received vaccination (online Technical Appendix). The reproduction number within a ring was therefore reduced by a factor of $1-(0.8 \times 0.7)=0.44$ once the vaccine became effective (online Technical Appendix Figure 3).

To estimate the effect of ring vaccination, we simulated multiple outbreaks and calculated the proportion of these outbreaks that became large (i.e., $>500$ clusters). We found that if more than a few cases were missed, large outbreaks could occur under ring vaccination (Figure 2, panel A). This event could occur because missed cases, which had a higher reproduction number, would not be inside the ring when vaccination was introduced. Although ring vaccination failed to contain the outbreak in this scenario, it still reduced disease transmission (online Technical Appendix 

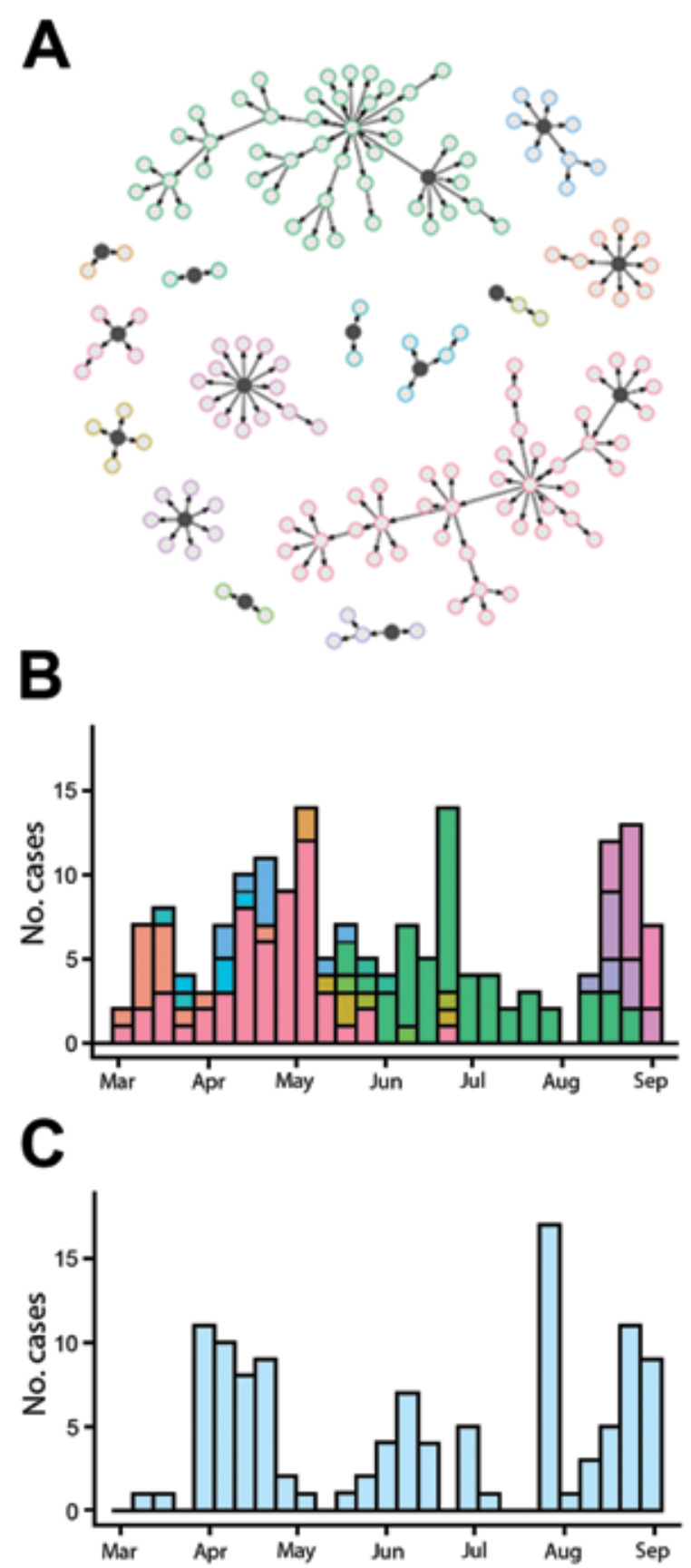

Figure 1. Outbreak dynamics in a model of transmission of Ebola virus disease. A) Chains of transmission generated in a simulated outbreak starting with 2 infected persons on March 1, 2014. Black circles indicate the index case within each cluster, and arrows indicate routes of transmission. Within each cluster, we assumed that there was a $15 \%$ probability that a secondary case would be missed and would instead seed a new cluster (these missed links are not shown). B) New cases per week, by date of symptom onset, for the chains of transmission shown in panel A. Colors of clusters in panel A match colors of bars in panel B. C) Observed weekly confirmed and probable cases reported in Conakry Prefecture, Guinea, during March-September 2014. Data were obtained from the Guinea Ministry of Health and World Health Organization Situation Reports (11).

Figure 4). We also considered the effect of preemptive mass vaccination, which reduced the reproduction number for all cases by a factor of 0.44 , regardless of whether cases were in the cluster or missed. This strategy was more effective in containing outbreaks, even if many cases were missed (Figure 2, panel A). Similar qualitative patterns were observed when vaccine efficacy was 95\% (online Technical Appendix Figure 5).

In the later stages of the EVD epidemic in West Africa, behavior changes and improved control measures led to less transmission from burials and in hospital settings than in early 2014 (12). Similar reductions were observed in other Ebola outbreaks (e.g., in 1976 in Yambuku, Zaire) (13). We therefore also explored a partial control scenario. We omitted index cases in the 2014 Guinea transmission chains that were involved in funeral or hospital transmission, which resulted in $R_{m}=2.5$ for missed cases (online Technical Appendix Figure 6). We also assumed a shorter duration of infectiousness and time to reporting on the basis of data for 2015 $(3,10)$ (online Technical Appendix Table 2).

In this partial control scenario, outbreaks could be controlled with ring vaccination, even if $40 \%$ of cases were missed (Figure 2, panel B). Our results suggest that ring vaccination could substantially reduce the potential size and duration of outbreaks if other control measures are also in place (Table). We also estimated how many vaccine doses would be required for ring vaccination (online Technical Appendix); in the partial control scenario, several thousand doses might be needed (online Technical Appendix Table 3 ). We could not estimate doses required for mass vaccination, and thus could not perform an economic analysis of different strategies, because this would depend on the potential for long-distance transmission events and populations in different areas. However, implementing mass vaccination for even a single district in West Africa could require $>100,000$ doses.

Our analysis has some limitations. In the early 2014 transmission scenario, we assumed that missed cases had a much higher reproduction number than cases within clusters. However, if an effective vaccine became available, persons at risk might be more likely to engage with public health efforts. The high reproduction number for index cases might also be caused in part by ascertainment bias: cases that generate many secondary infections are more likely to be designated as index cases. We also assumed that mass vaccination would target $70 \%$ of the population at random; in practice, there could be clustering effects. Furthermore, we assumed that chains of transmission were independent and that the reproduction number remained unchanged over time. In reality, missed cases might have shared contacts and behavior might change during outbreak, which could reduce transmission. Our estimates are therefore likely to represent a reasonable worst-case scenario. 

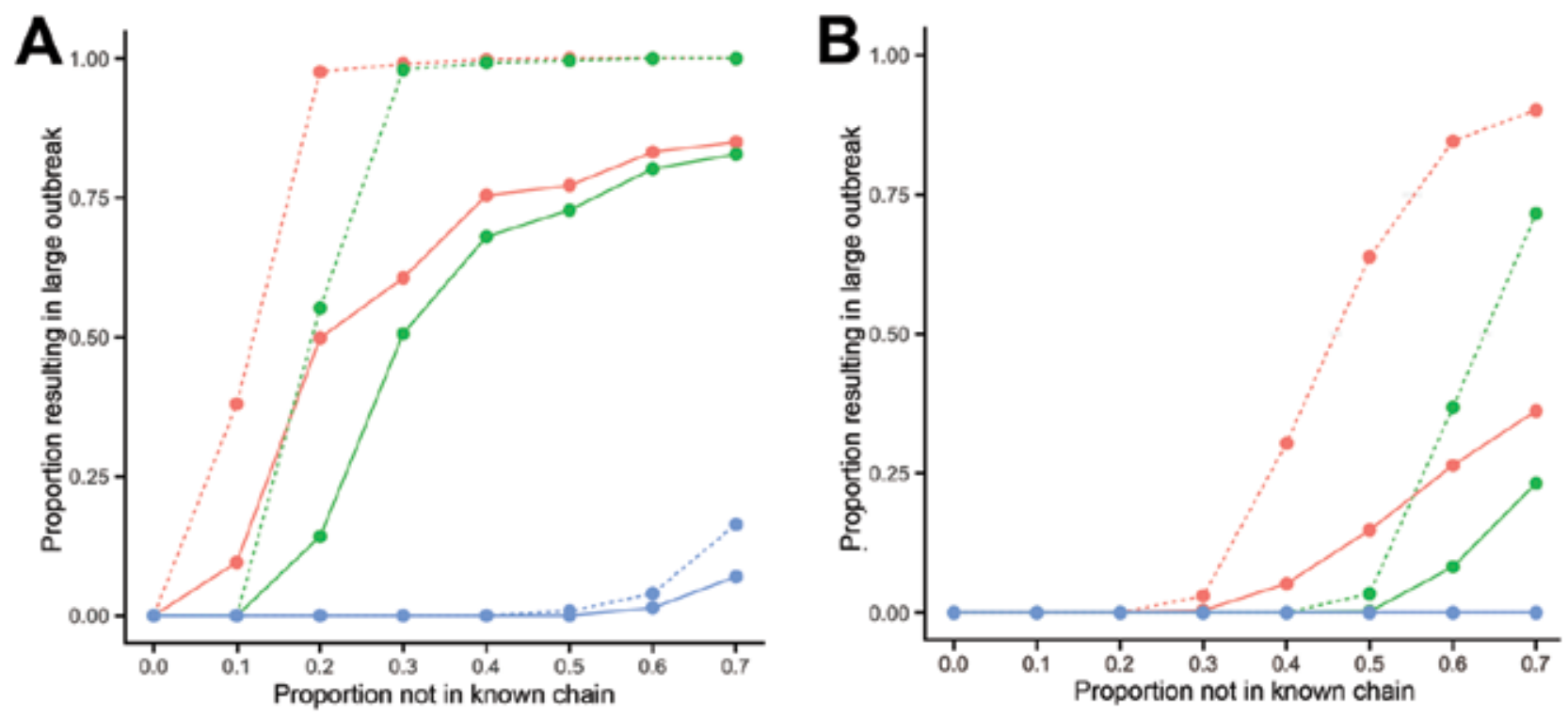

Figure 2. Effectiveness of vaccination strategies for Ebola virus disease under different transmission scenarios. A) Proportion of simulations that led to a large outbreak (defined as $>500$ clusters) in the early 2014 Guinea transmission scenario. Red lines indicate no vaccination, green lines indicate ring vaccination, blue lines indicate mass vaccination; solid lines indicate outbreaks that started with 1 index case, and dashed lines indicate outbreaks that started with 5 index cases. We simulated 1,000 outbreaks and calculated the proportion that resulted in $>500$ clusters. When the space between the red and green lines is large, the model suggests that ring vaccination would provide substantial additional value over standard public health control measures alone. B) Proportion of simulations that led to a large outbreak in partial control scenario.

\section{Conclusions}

Ring vaccination enhances standard public health measures of contact tracing, isolation, and community engagement (14) and could be effective when such measures are in place. However, if standard measures are not working because many cases are not in known transmission chains, as in West Africa in early 2014, ring vaccination might be insufficient to contain the outbreak. If an EVD vaccine is shown to be efficacious, our results suggest that mass vaccination, or hybrid strategies involving mass and ring vaccinations, might need to be considered alongside ring vaccination when planning for future outbreaks.
This study was supported by the Research for Health in Humanitarian Crises Programme, which is managed by Research for Humanitarian Assistance (grant 13165). C.H.W. was supported by the Medical Research Council (grant MR/J003999/1) and the Norwegian Institute of Public Health. R.M.E. and W.J.E. were supported by Innovative Medicines Initiative 2 (IMI2) Joint Undertaking under grant agreement EBOVAC1 (grant 115854). The IMI2 is supported by the European Union Horizon 2020 Research and Innovation Programme and the European Federation of Pharmaceutical Industries and Associations.

Dr. Kucharski is a lecturer in infectious disease epidemiology at the London School of Hygiene and Tropical Medicine, London, UK. His research interests are transmission and control of emerging infectious diseases.

\begin{tabular}{|c|c|c|c|}
\hline Probability of case missed & No vaccination & Ring vaccination & Mass vaccination \\
\hline \multicolumn{4}{|l|}{ Median no. cases $(95 \% \mathrm{Cl})$} \\
\hline $10 \%$ & $42(14-235)$ & $30(13-79)$ & $13(7-60)$ \\
\hline $20 \%$ & $63(15-551)$ & $39(14-131)$ & $13(7-57)$ \\
\hline $30 \%$ & $104(17-2,660)$ & $53(15-229)$ & $13(6-48)$ \\
\hline $40 \%$ & $296(20-2,410)$ & $78(18-452)$ & $13(6-46)$ \\
\hline \multicolumn{4}{|c|}{ Duration of outbreak, $\mathrm{d}(95 \% \mathrm{Cl})$} \\
\hline $10 \%$ & 87 (28-278) & $62(26-145)$ & $41(12-139)$ \\
\hline $20 \%$ & $123(33-480)$ & $83(31-214)$ & $43(11-149)$ \\
\hline $30 \%$ & $185(43-1,020)$ & $110(36-319)$ & $47(11-142)$ \\
\hline $40 \%$ & $364(51-1,150)$ & $149(45-486)$ & $47(9-147)$ \\
\hline
\end{tabular}




\section{References}

1. Wellcome Trust, Center for Infectious Disease Research and Policy. Recommendations for accelerating the development of Ebola vaccines [cited 2015 Oct 20]. http://www.wellcome.ac.uk/ stellent/groups/corporatesite/@policy_communications/documents/ web_document/wtp058693.pdf

2. Ebola ça Suffit Ring Vaccination Trial Consortium. The ring vaccination trial: a novel cluster randomised controlled trial design to evaluate vaccine efficacy and effectiveness during outbreaks, with special reference to Ebola. BMJ. 2015;351:h3740.

3. Henao-Restrepo AM, Longini I Jr, Egger M, Dean NE, Edmunds WJ, Camacho A, et al. Efficacy and effectiveness of an rVSV-vectored vaccine expressing Ebola surface glycoprotein: interim results from the Guinea ring vaccination cluster-randomised trial. Lancet. 2015;386:857-66.

4. Fenner F, Henderson DA, Arita I, Jezek Z, Ladnyi ID. Smallpox and its eradication. Geneva: World Health Organization; 1988.

5. Wells C, Yamin D, Ndeffo-Mbah ML, Wenzel N, Gaffney SG, Townsend JP, et al. Harnessing case isolation and ring vaccination to control Ebola. PLoS Negl Trop Dis. 2015;9:e0003794. http://dx.doi.org/10.1371/journal.pntd.0003794

6. Althaus CL. Ebola superspreading. Lancet Infect Dis. 2015;15:507-8. http://dx.doi.org/10.1016/S1473-3099(15)70135-0

7. Faye O, Böelle PY, Heleze E, Faye O, Loucoubar C, Magassouba N, et al. Chains of transmission and control of Ebola virus disease in Conakry, Guinea, in 2014: an observational study. Lancet Infect Dis. 2015;15:320-6. http://dx.doi.org/10.1016/ S1473-3099(14)71075-8
8. Kretzschmar M, Van den Hof S, Wallinga J, Van Wijngaarden J. Ring vaccination and smallpox control. Emerg Infect Dis. 2004;10:832-41. http://dx.doi.org/10.3201/eid1005.030419

9. Lloyd-Smith JO, Schreiber SJ, Kopp PE, Getz WM. Superspreading and the effect of individual variation on disease emergence. Nature. 2005;438:355-9. http://dx.doi.org/10.1038/ nature 04153

10. WHO Ebola Response Team. Ebola virus disease in West Africathe first 9 months of the epidemic and forward projections. N Engl J Med. 2014;371:1481-95. http://dx.doi.org/10.1056/NEJMoa1411100

11. Centre for the Mathematical Modelling of Infectious Diseases. Visualisation and projections of the Ebola outbreak in West Africa [cited 2015 Oct 20]. http://cmmid.lshtm.ac.uk/ebola/

12. Nielsen CF, Kidd S, Sillah AR, Davis E, Mermin J, Kilmarx PH. Improving burial practices and cemetery management during an Ebola virus disease epidemic - Sierra Leone, 2014. MMWR Morb Mortal Wkly Rep. 2015;64:20-7.

13. Camacho A, Kucharski AJ, Funk S, Breman J, Piot P, Edmunds WJ. Potential for large outbreaks of Ebola virus disease. Epidemics. 2014;9:70-8. http://dx.doi.org/10.1016/j.epidem.2014.09.003

14. Heymann DL. Ebola: learn from the past. Nature. 2014;514:299300. http://dx.doi.org/10.1038/514299a

Address for correspondence: Adam Kucharski, Department of Infectious Disease Epidemiology, London School of Hygiene and Tropical Medicine, London WC1E 7HT, UK; email: adam.kucharski@1shtm.ac.uk

\section{August 2015: Surveillance Including:}

- Escherichia coli O157 Outbreaks in the United States, 2003-2012

- Underrecognition of Dengue during 2013 Epidemic in Luanda, Angola

- Health Care-Associated Infection Outbreak Investigations in Outpatient Settings, Los Angeles County, California, USA, 2000-2012

- Differentiation of Acute Q Fever from Other Infections in Patients Presenting to Hospitals, the Netherlands

- Community-Based Outbreak of Neisseria meningitidis Serogroup C Infection in Men who Have Sex with Men, New York City, New York, USA, 2010-2013

- Risk for Mycobacterial Disease among Patients with Rheumatoid Arthritis, Taiwan, 2001-2011

- Phylogeography of Influenza A(H3N2) Virus in Peru, 2010-2012

- Susceptibility of Carrion Crows to Experimental Infection with Lineage 1 and 2 West Nile Viruses

- Influenza A Viruses of Human Origin in Swine, Brazil

http://wwwnc.cdc.gov/eid/articles/issue/21/08/table-of-contents 